\title{
Binding thermodynamics of host-guest systems with SMIRNOFF99Frosst 1.0 .5 from the Open Force Field Initiative
}

David R. Slochower ${ }^{1}$, Niel M. Henriksen ${ }^{1}$, Lee-Ping Wang'2, John D. Chodera ${ }^{3}$, David L. Mobley4, and Michael K. Gilson ${ }^{1}$

${ }^{1}$ Skaggs School of Pharmacy and Pharmaceutical Sciences, University of California, San Diego, La Jolla, CA 92093, USA

2 Department of Chemistry, University of California, Davis, CA 95616, USA

${ }^{3}$ Computational and Systems Biology Program, Sloan Kettering Institute, Memorial Sloan

Kettering Cancer Center, New York, NY 10065

4 Department of Pharmaceutical Sciences and Department of Chemistry, University of California, Irvine, CA 92697, USA

A

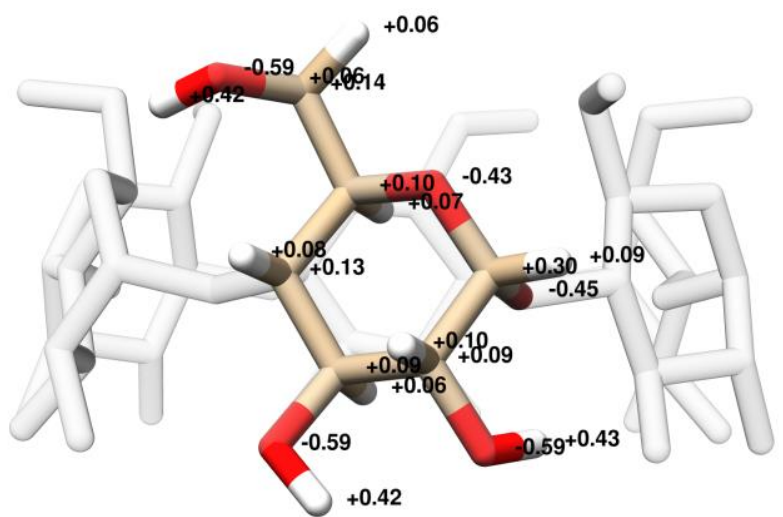

B

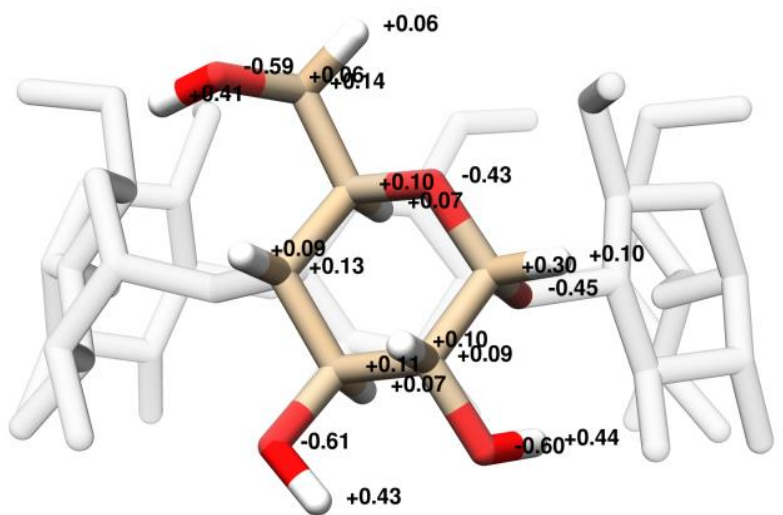

Figure S1: Comparison of AM1-BCC partial atomic charges assigned by running antechamber on a single glucose monomer (A) or on an entire $\alpha C D$ molecule (B) with the option -pl 10 to specify the maximum path length used to determine the equivalence of atomic charges. 

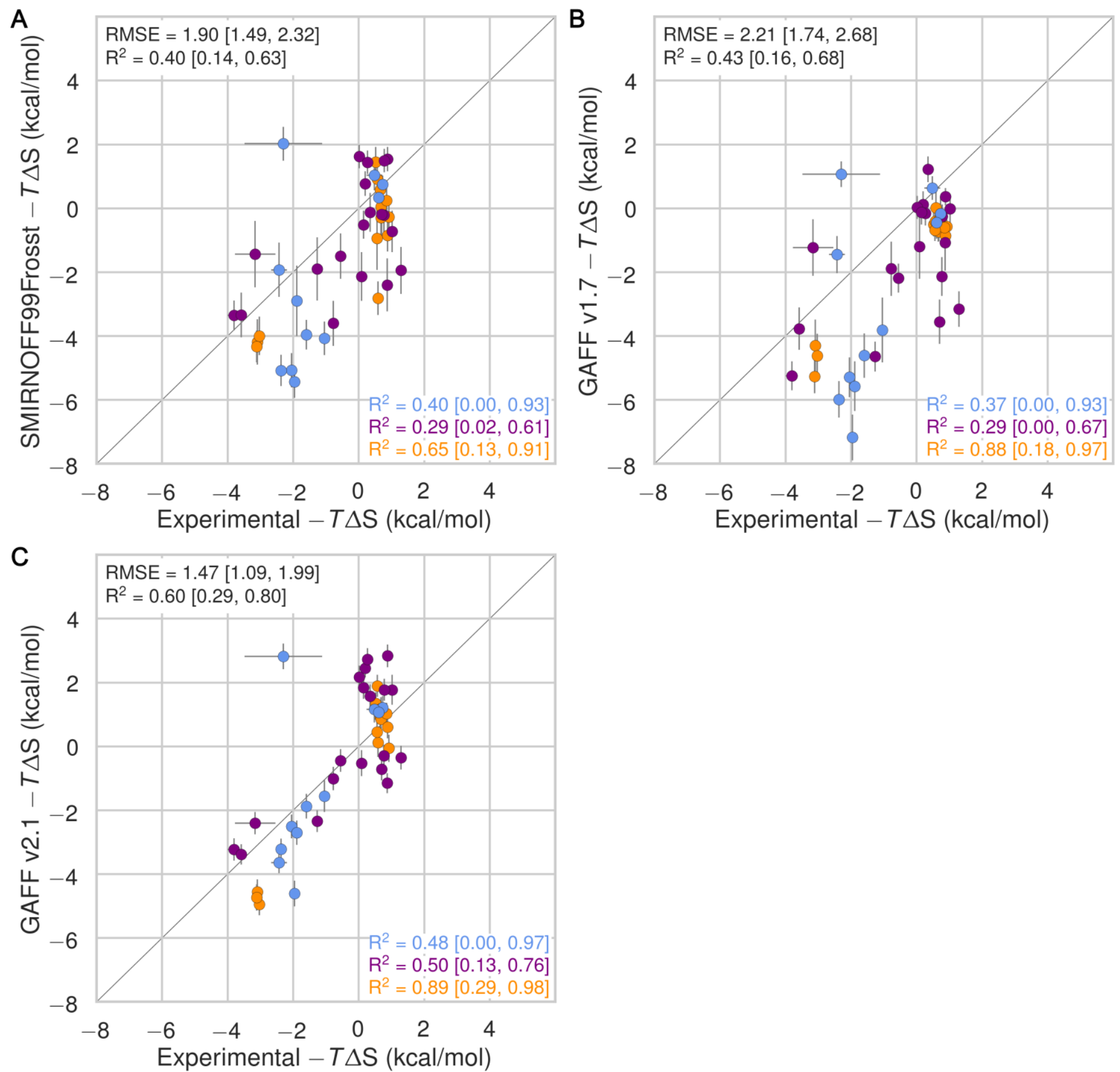

Figure S2: Comparison of calculated absolute binding entropies $\left(-\mathrm{T} \Delta \mathrm{S}^{\circ}\right)$ with experiment with SMIRNOFF99Frosst parameters (top), GAFF v1.7 parameters (middle), or GAFF v2.1 parameters (bottom) applied to both host and guest. The orange, blue, and purple coloring distinguish the functional group of the guest as an ammonium, alcohol, or carboxylate, respectively. 
Primary cavity of $\alpha C D$
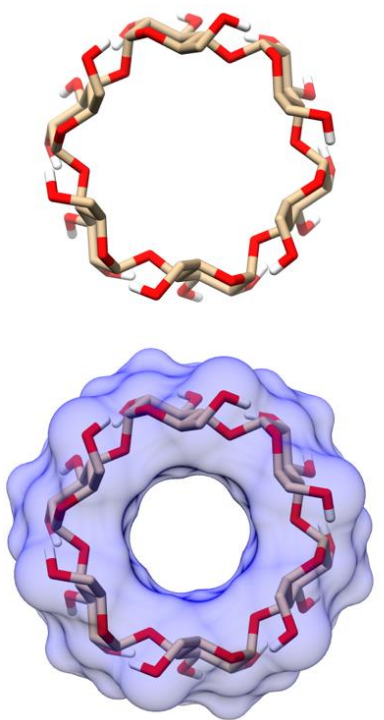

Secondary cavity of aCD
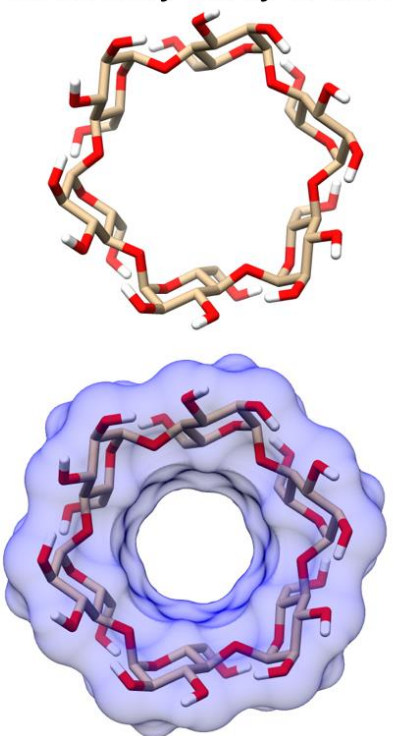

Figure S3: The primary (left) and secondary (right) face of $\alpha C D$.
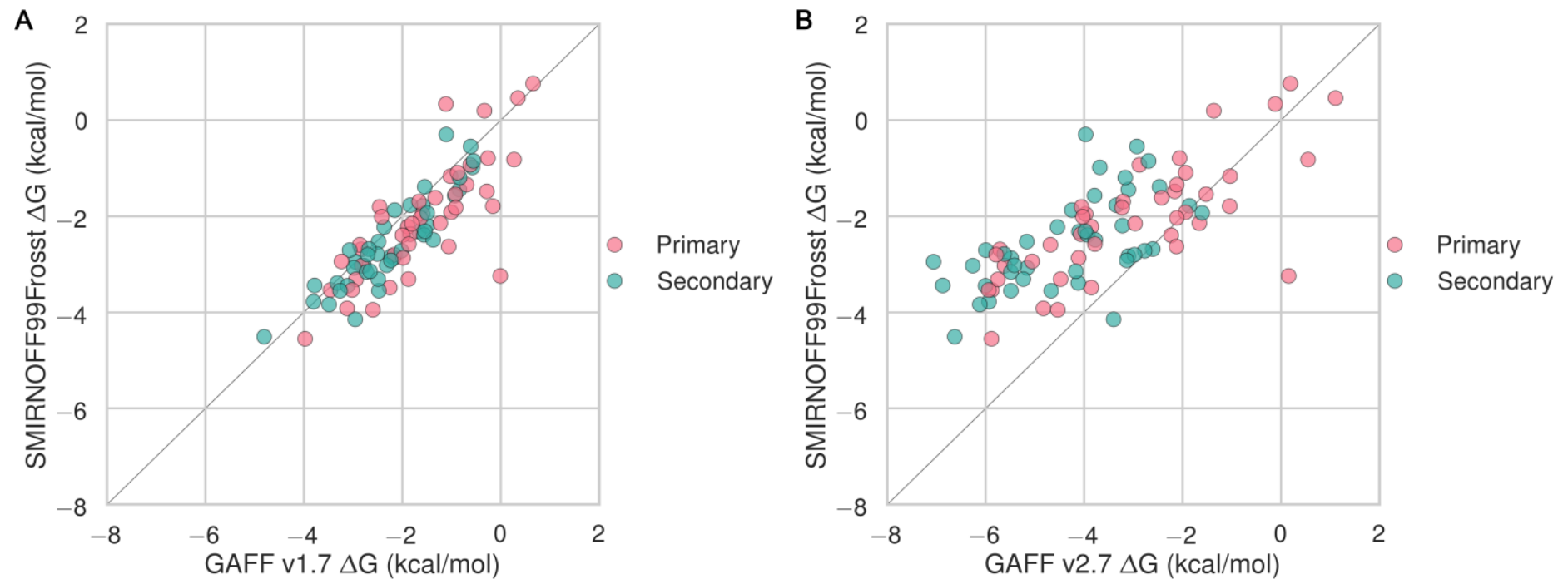

Figure S4: Binding free energies $\left(\Delta G^{\circ}\right)$ with the primary orientation results colored in blue and secondary orientation results colored in green. 

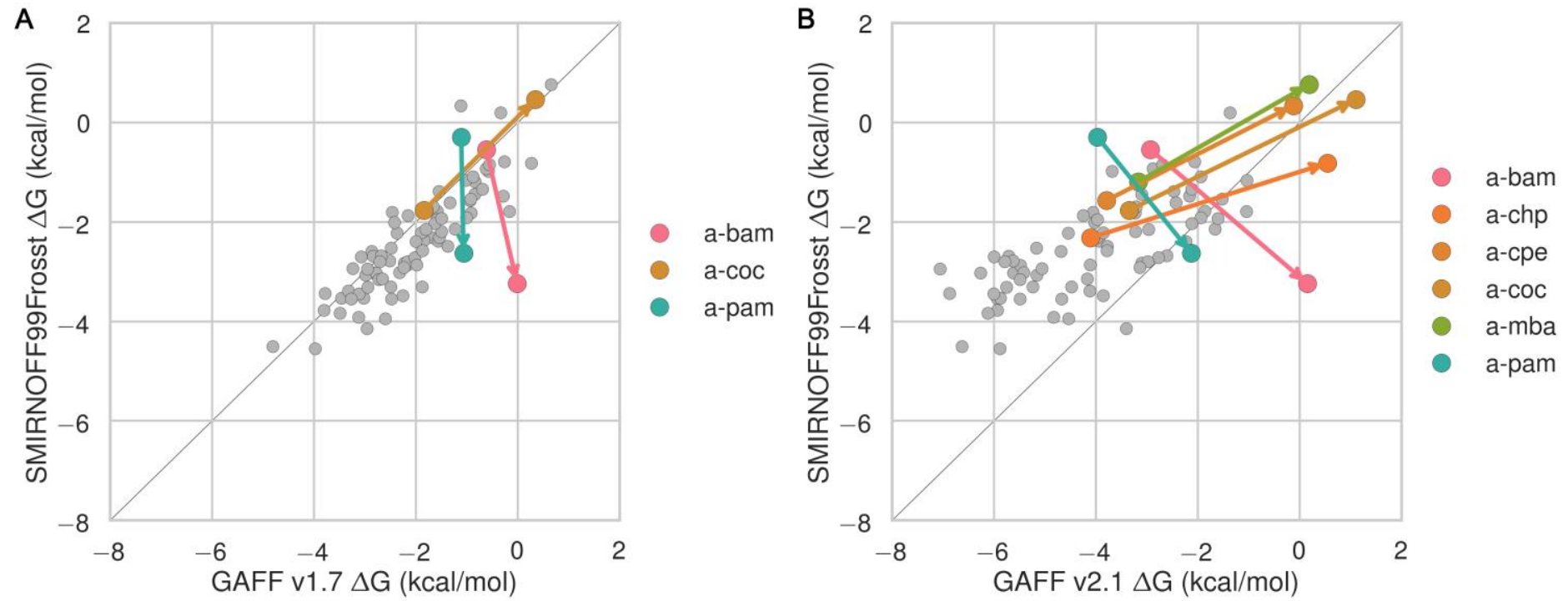

Figure S5: Binding free energies $\left(\Delta G^{\circ}\right)$ replotted from Figure S4 with points whose difference in binding free energy along either axis is greater than $2 \mathrm{kcal} / \mathrm{mol}$ shown in color. Arrows point from $\Delta \mathrm{G}^{\circ}$ for the secondary to $\Delta G^{\circ}$ for the primary cavity. 

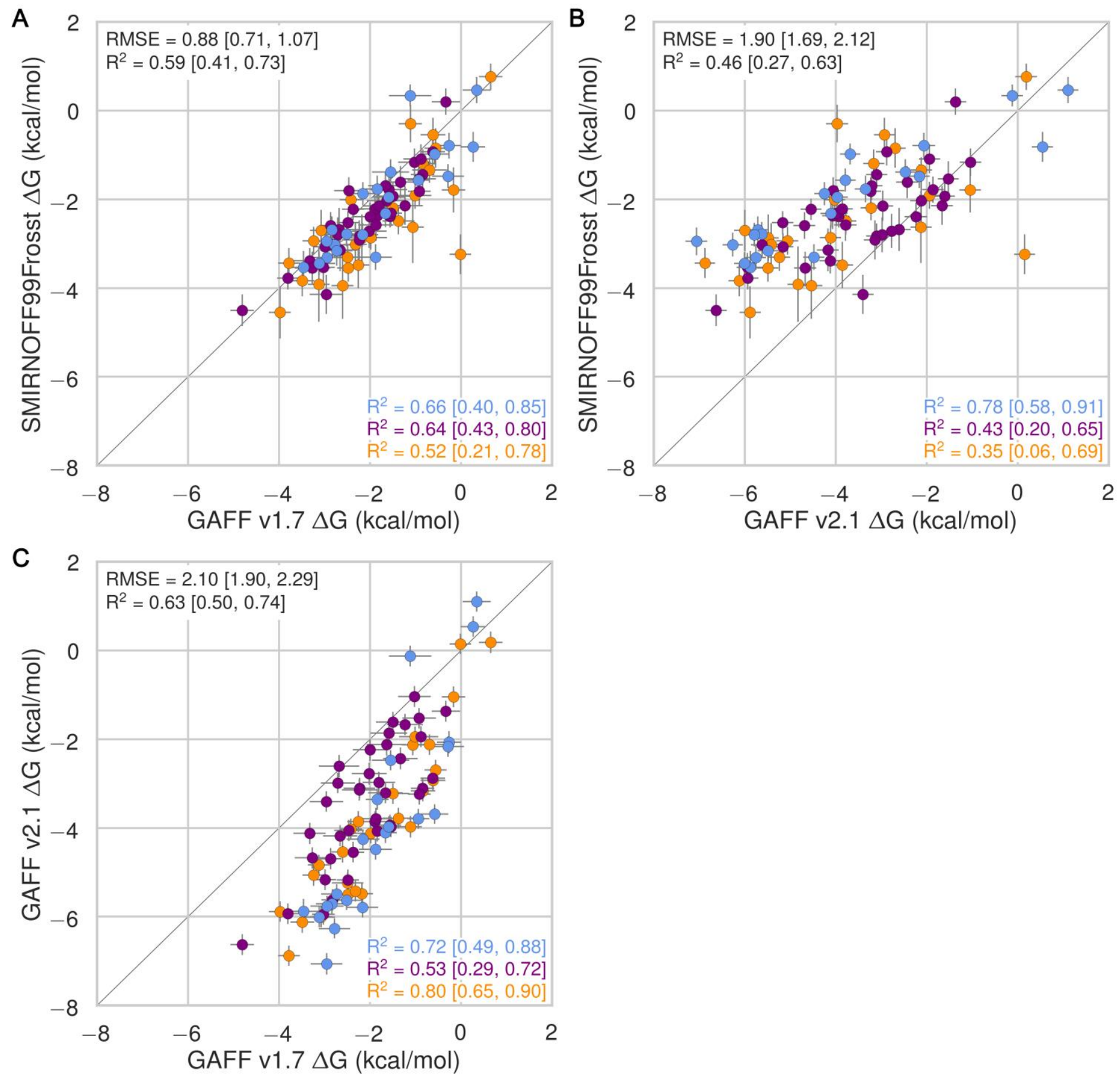

Figure S6: Comparison of calculated absolute binding free energies $\left(\Delta G^{\circ}\right)$ between force field combinations. The orange, blue, and purple coloring distinguish the functional group of the guest as an ammonium, alcohol, or carboxylate, respectively. 

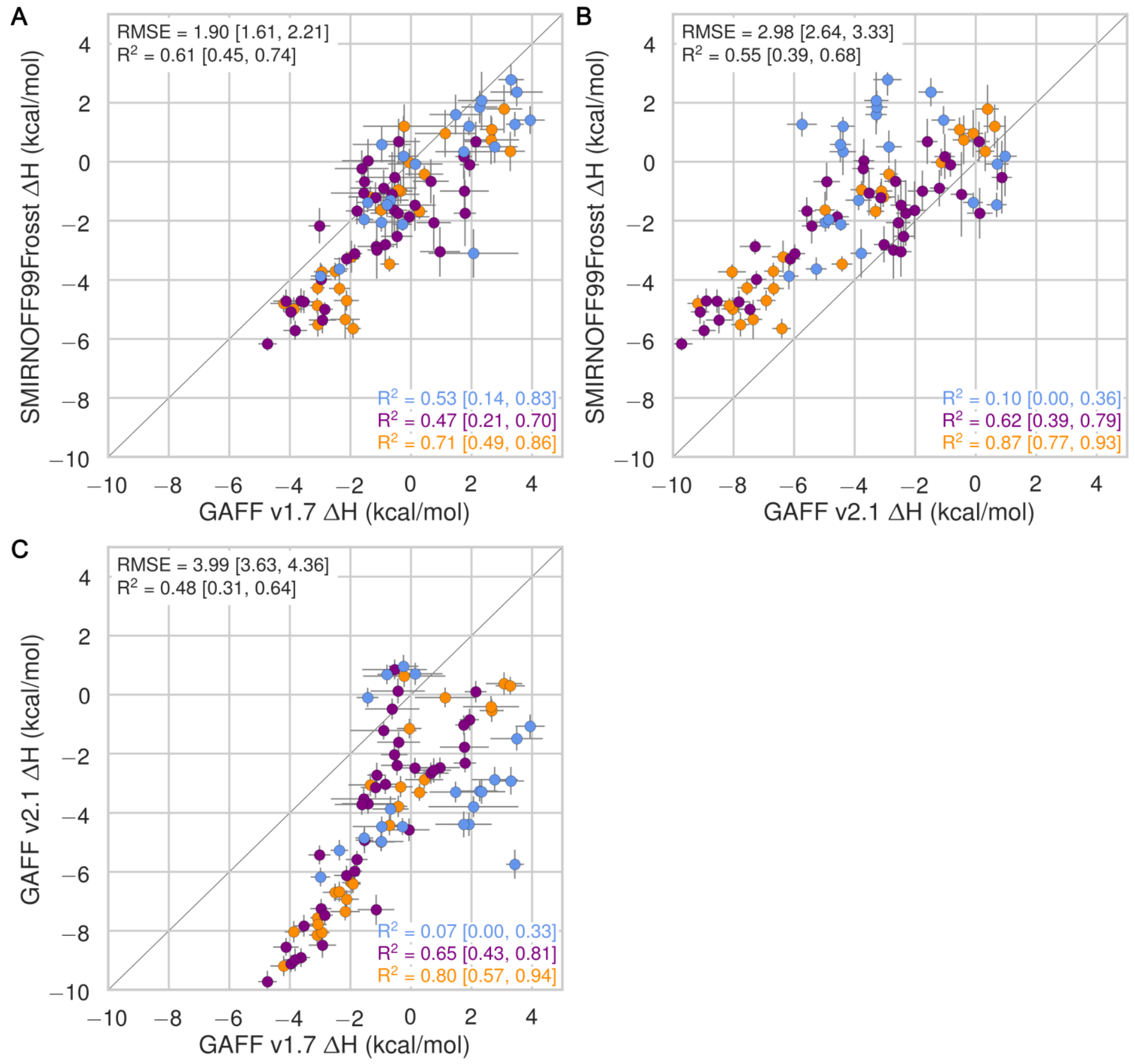

Figure S7: Comparison of calculated absolute binding free enthalpies $(\Delta \mathrm{H})$ between force field combinations. The orange, blue, and purple coloring distinguish the functional group of the guest as an ammonium, alcohol, or carboxylate, respectively. 

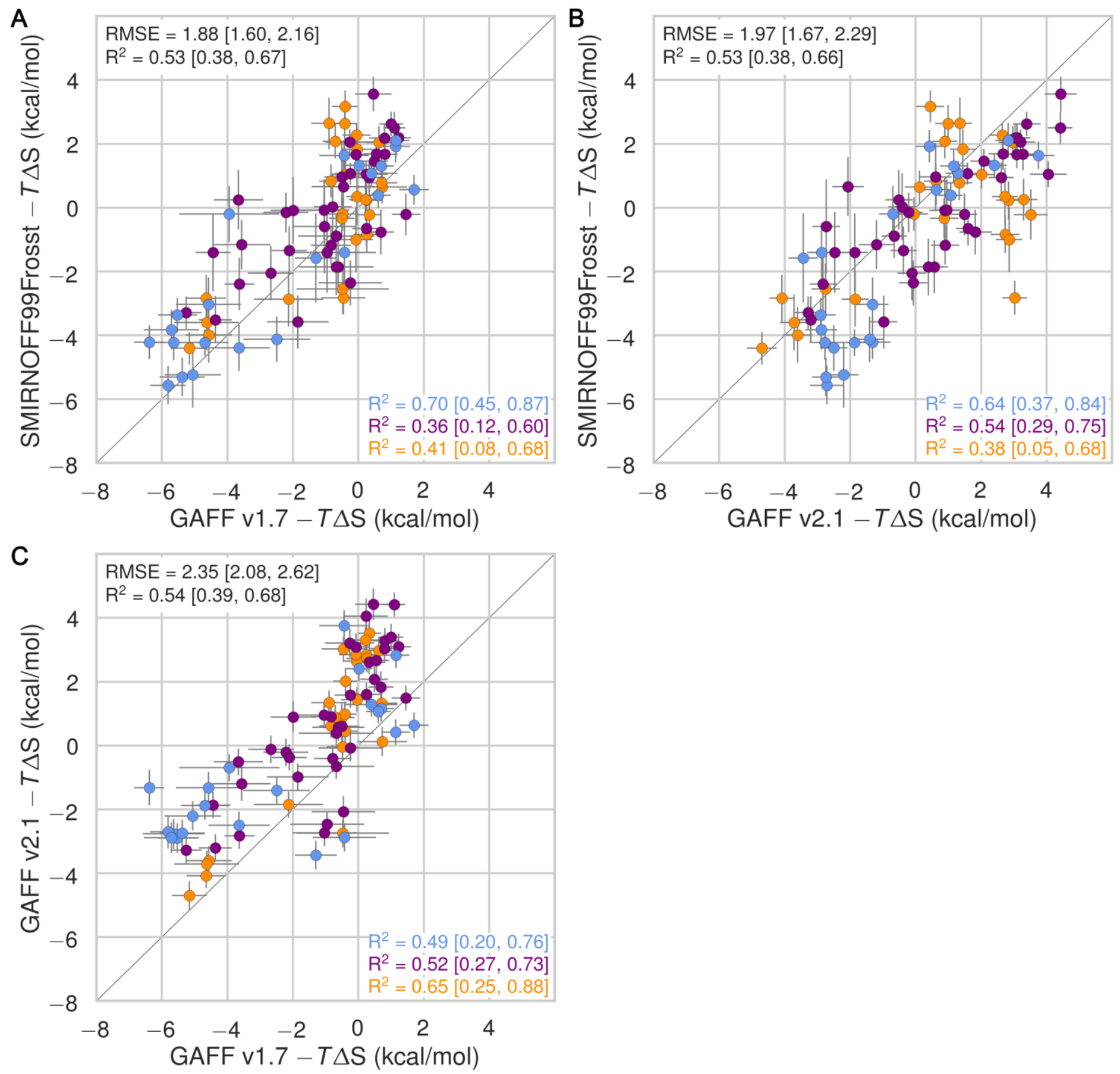

Figure S8: Comparison of calculated absolute binding entropies $\left(-\mathrm{T} \Delta \mathrm{S}^{\circ}\right)$ between force field combinations. The orange, blue, and purple coloring distinguish the functional group of the guest as an ammonium, alcohol, or carboxylate, respectively. 

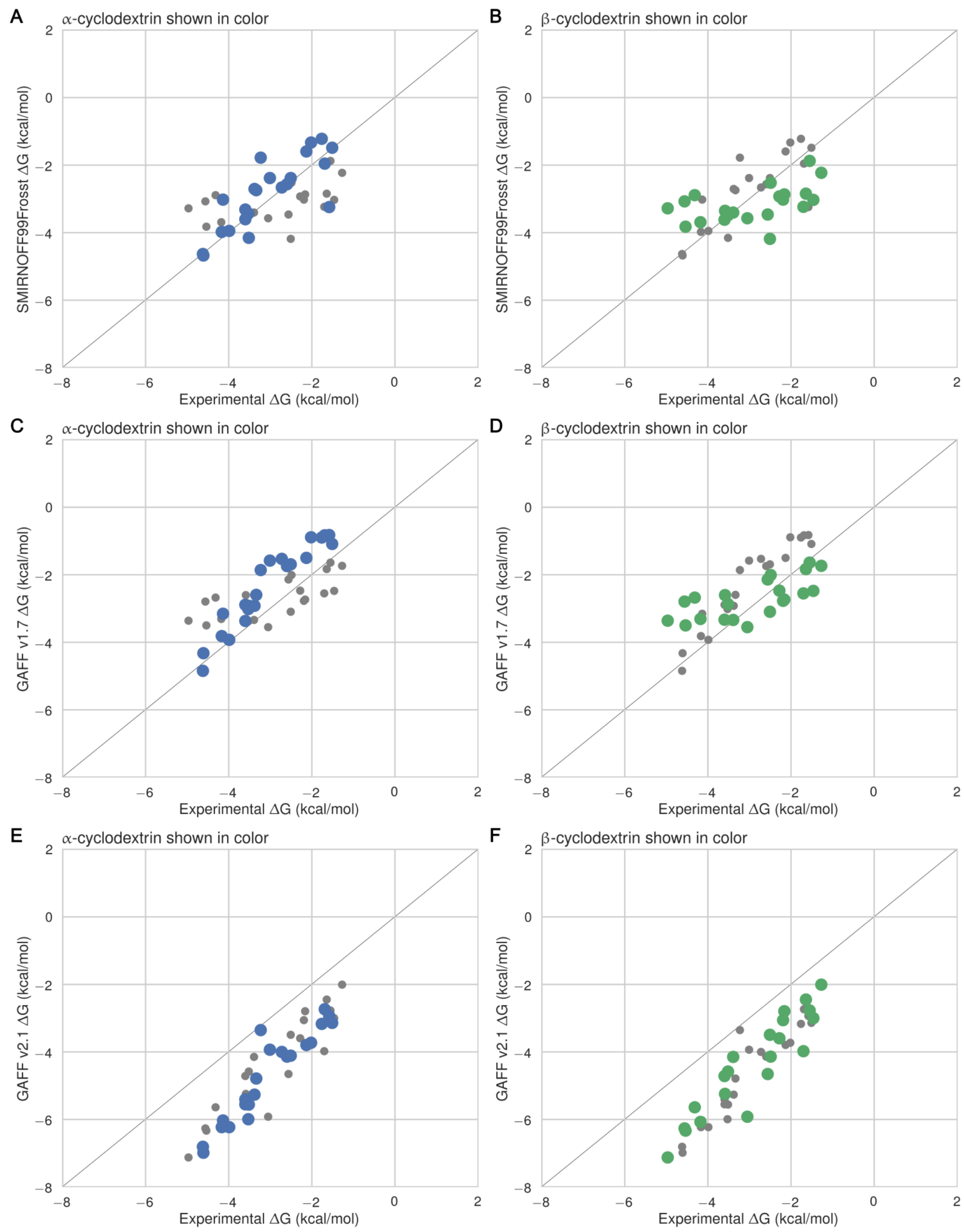
Figure S9: Binding free energies $\left(\Delta G^{\circ}\right)$ replotted from Figure 3, with $\alpha C D$ points colored in blue and $\beta C D$ points in grey (left) or $\alpha C D$ points in grey with $\beta C D$ points colored in green (right).
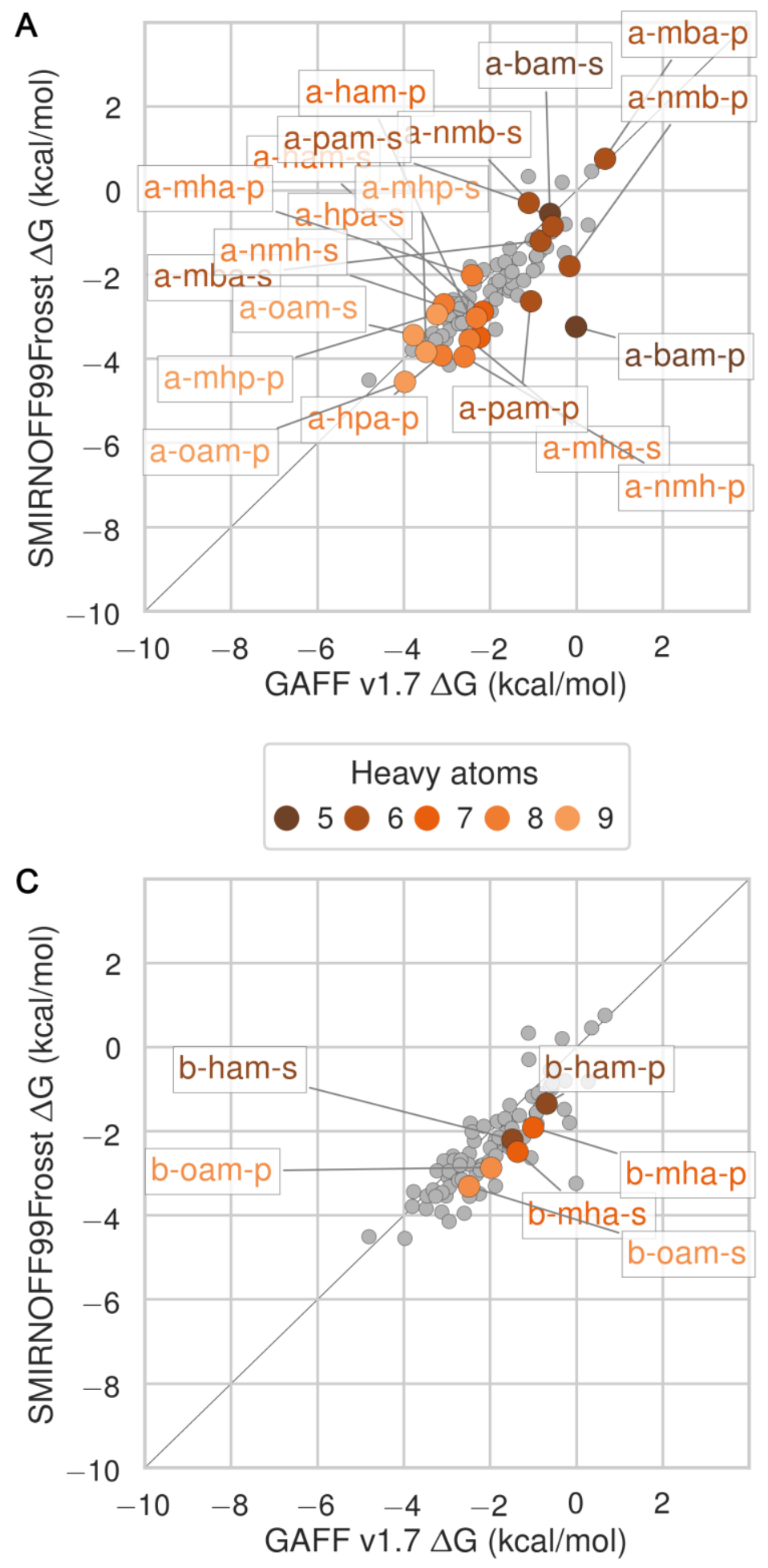

Heavy atoms

$7 \bigcirc 8 \bigcirc 9$

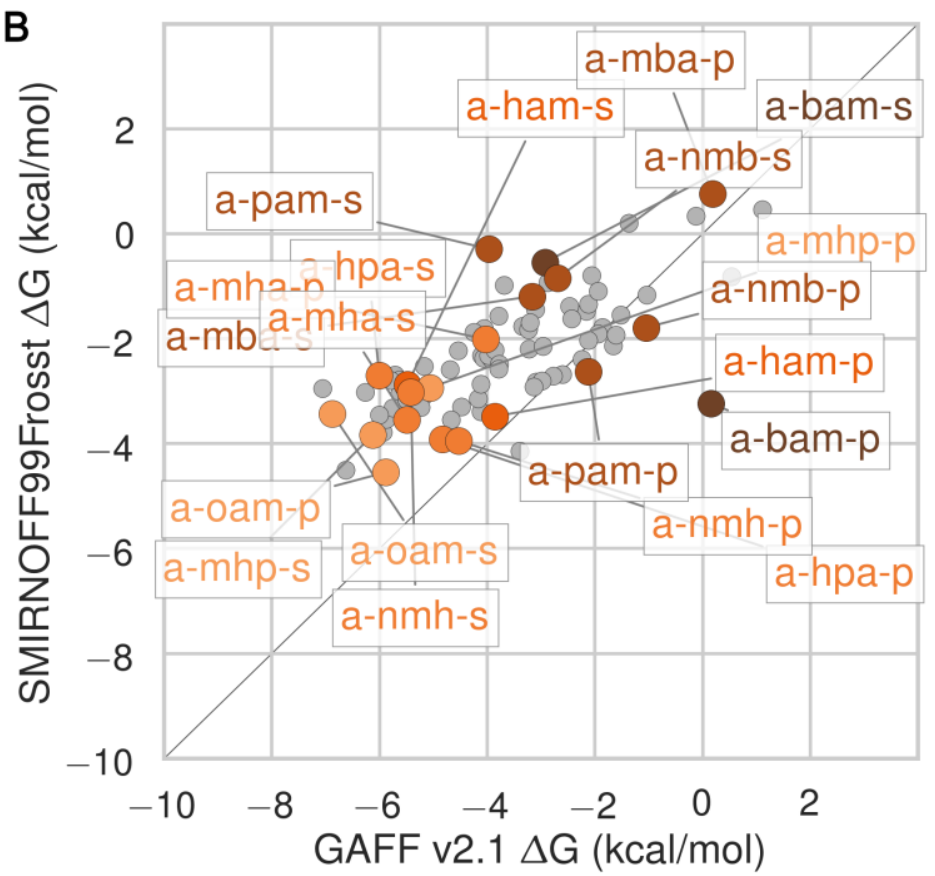

Heavy atoms

$5 \bigcirc 6 \bigcirc 7 \bigcirc 8 \bigcirc 9$

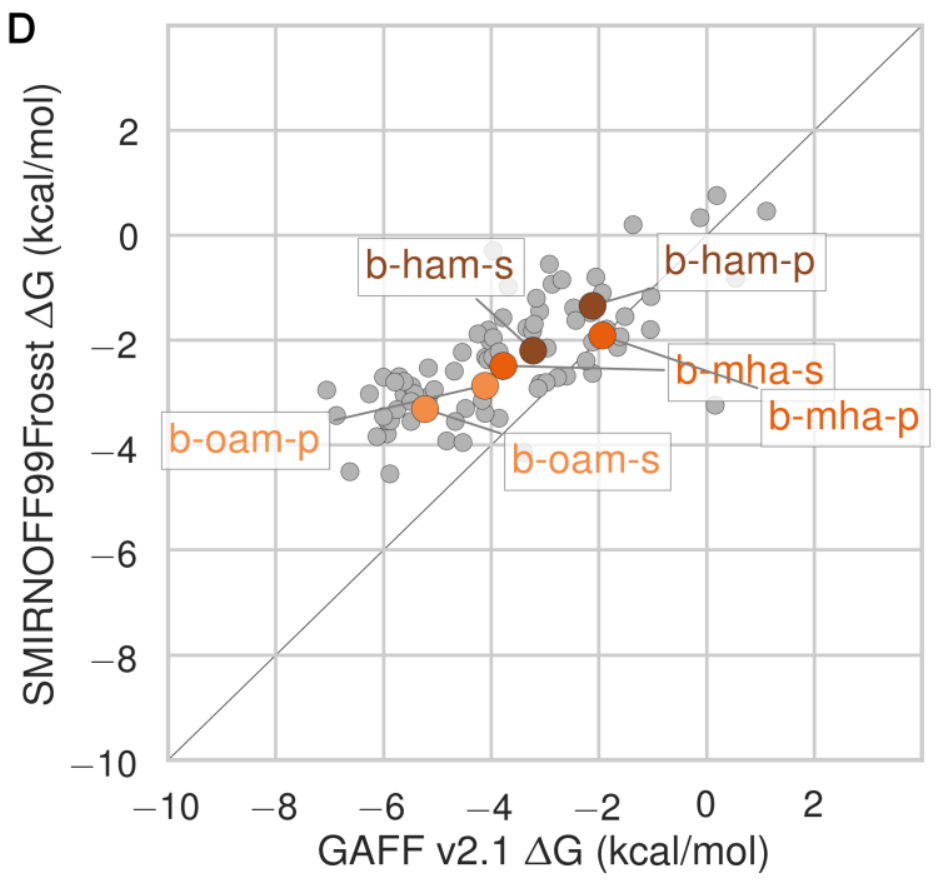

Heavy atoms

$7 \bigcirc 8 \bigcirc 9$

Figure S10: Binding free energy $\left(\Delta \mathrm{G}^{\circ}\right)$ comparisons showing ammonium guests in color and highlighted. 

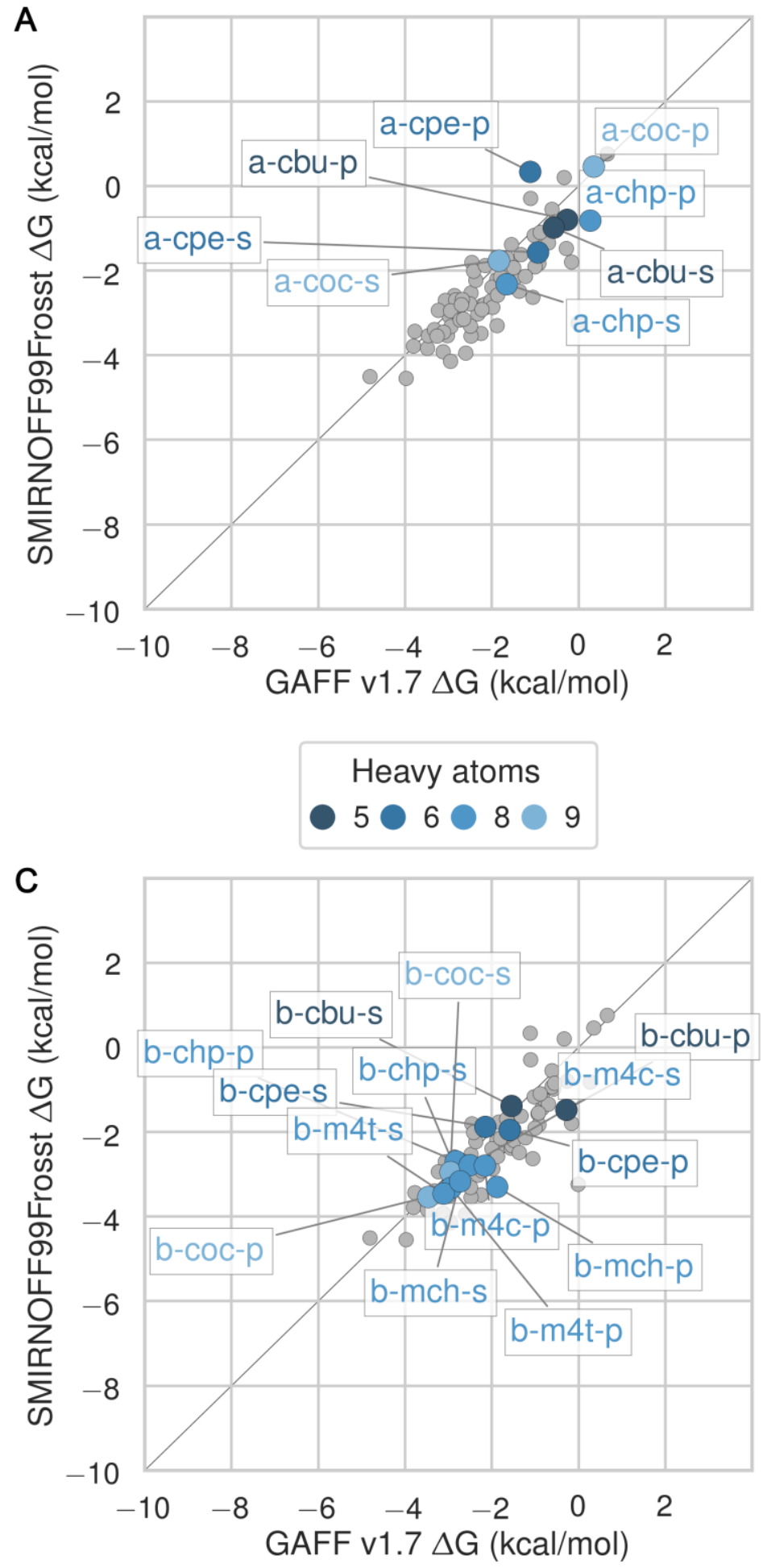

$$
\begin{aligned}
& \text { Heavy atoms } \\
& 5 \bigcirc 6 \bigcirc 8 \bigcirc 9
\end{aligned}
$$
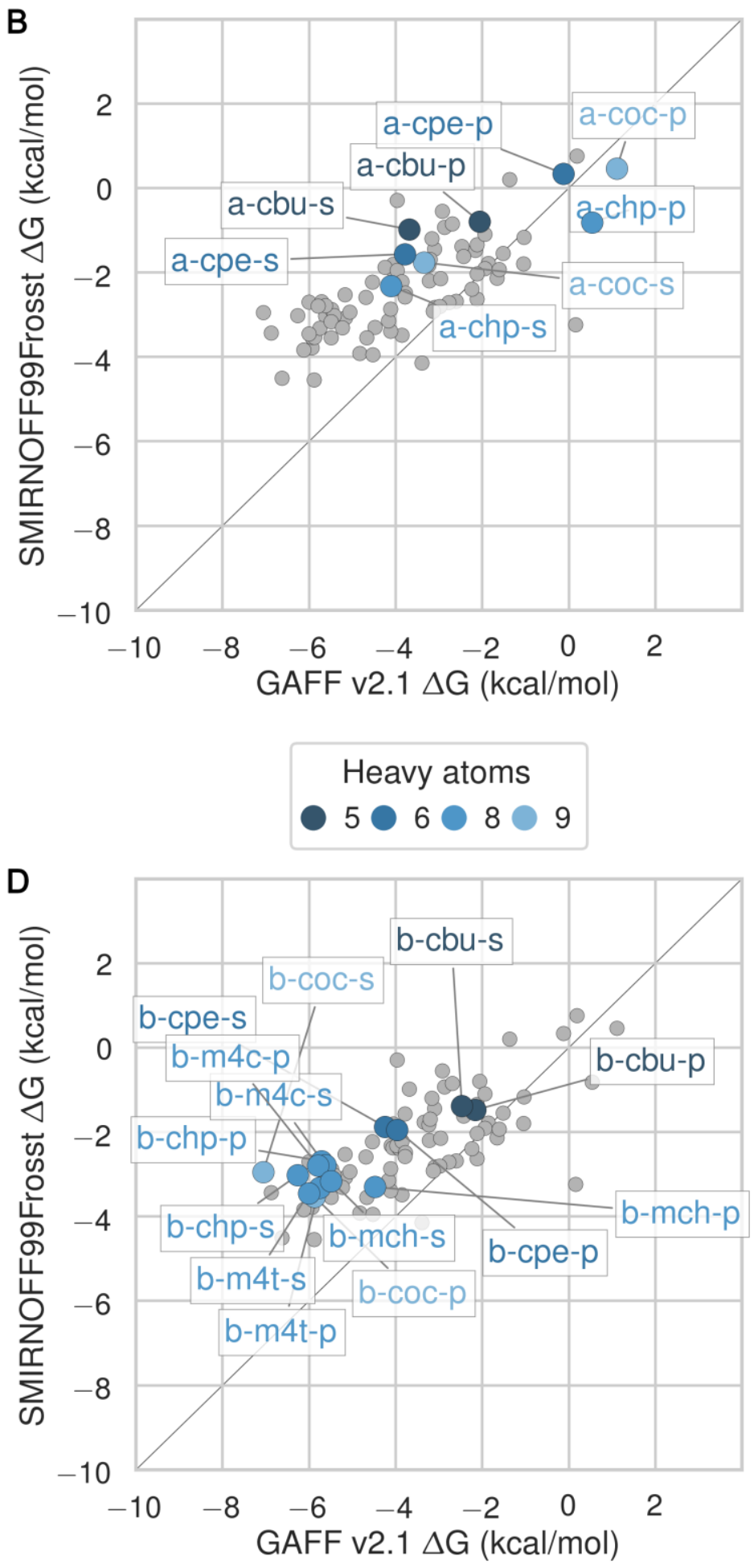

Heavy atoms

$5 \bigcirc 6 \bigcirc 8 \bigcirc 9$

Figure S11: Binding free energy $\left(\Delta \mathrm{G}^{\circ}\right)$ comparisons showing alcohols guests in color and highlighted. 

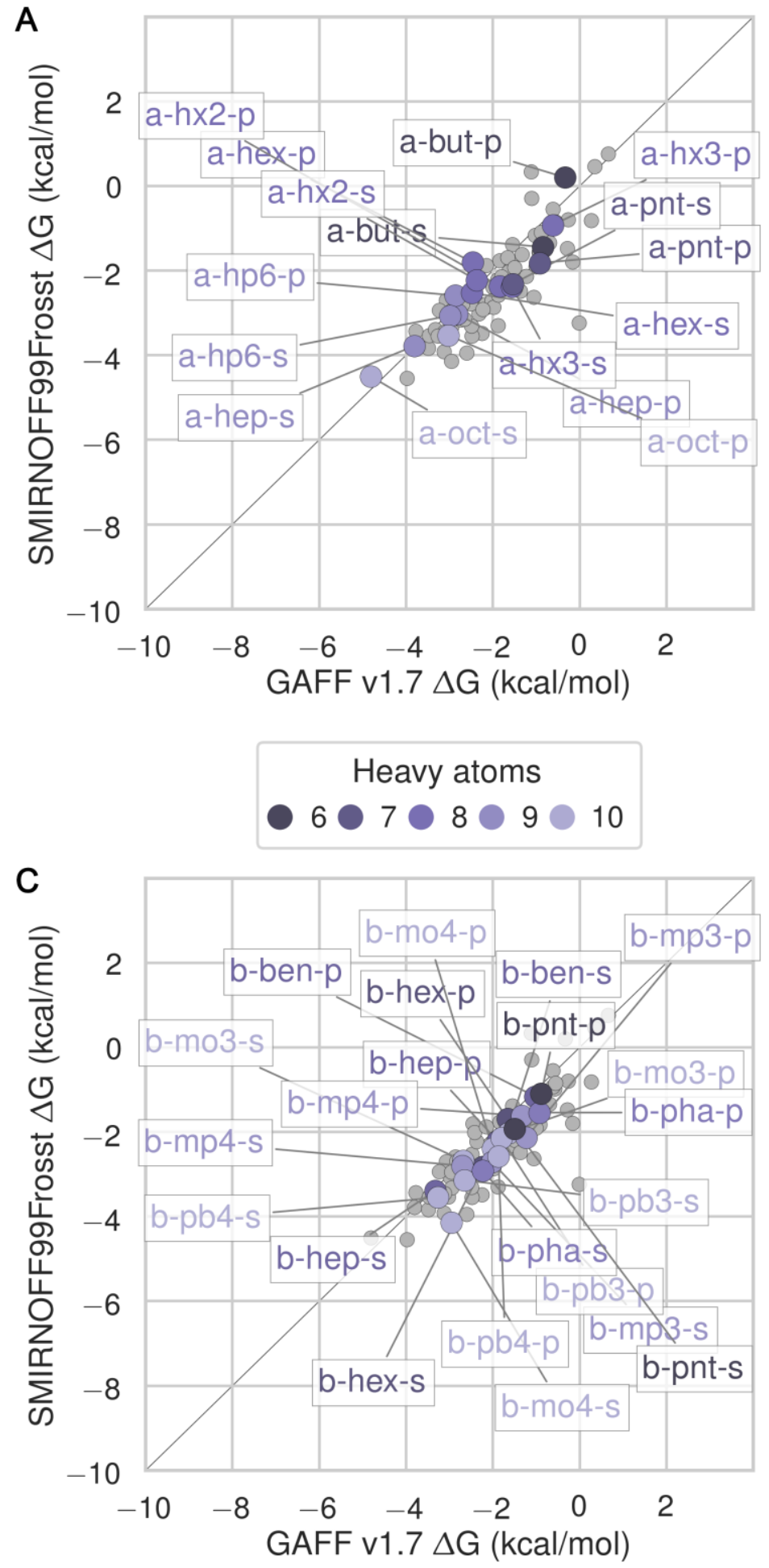

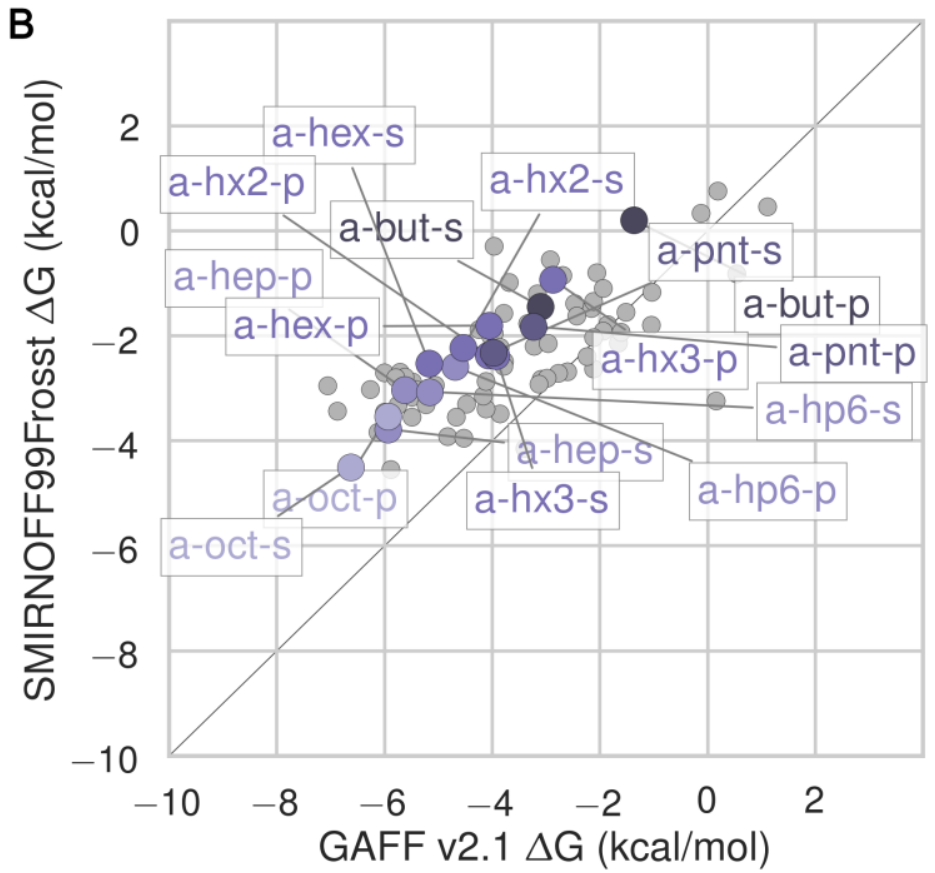

Heavy atoms

$6 \bigcirc 7 \bigcirc 8 \bigcirc 9 \bigcirc 10$

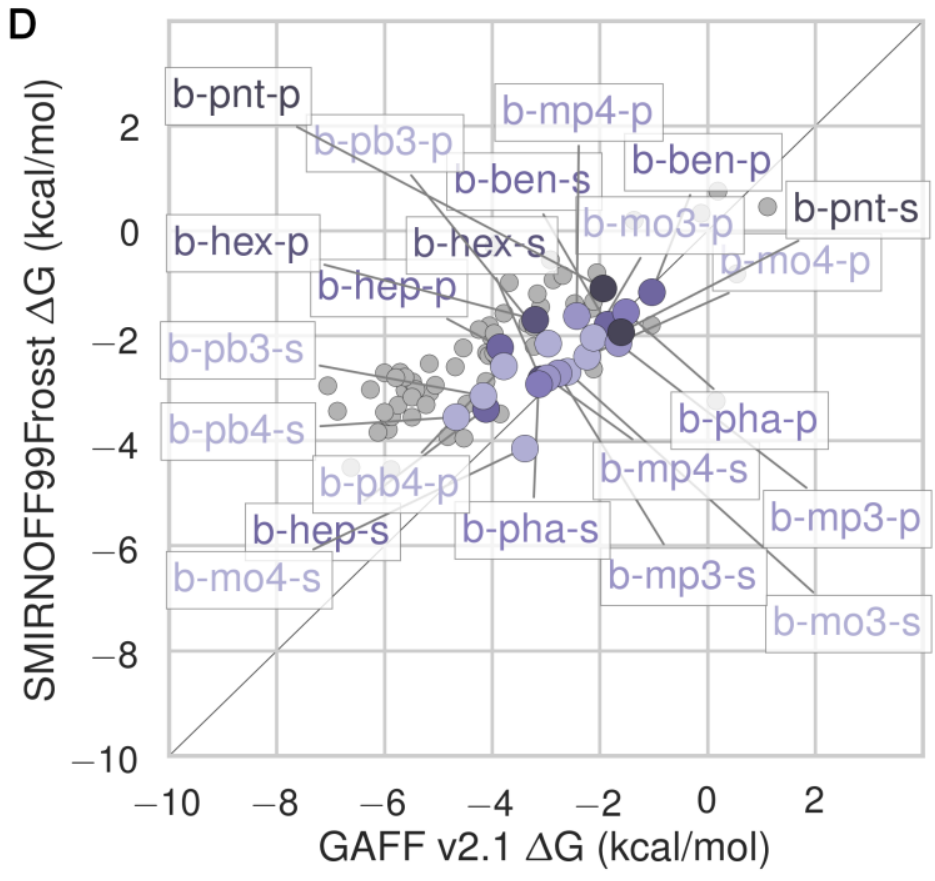

Heavy atoms

$7 \bigcirc 8 \bigcirc 9 \bigcirc 10 \bigcirc 11 \bigcirc 12$

Figure S12: Binding free energy $\left(\Delta \mathrm{G}^{\circ}\right)$ comparisons showing carboxylates guests in color and highlighted. 

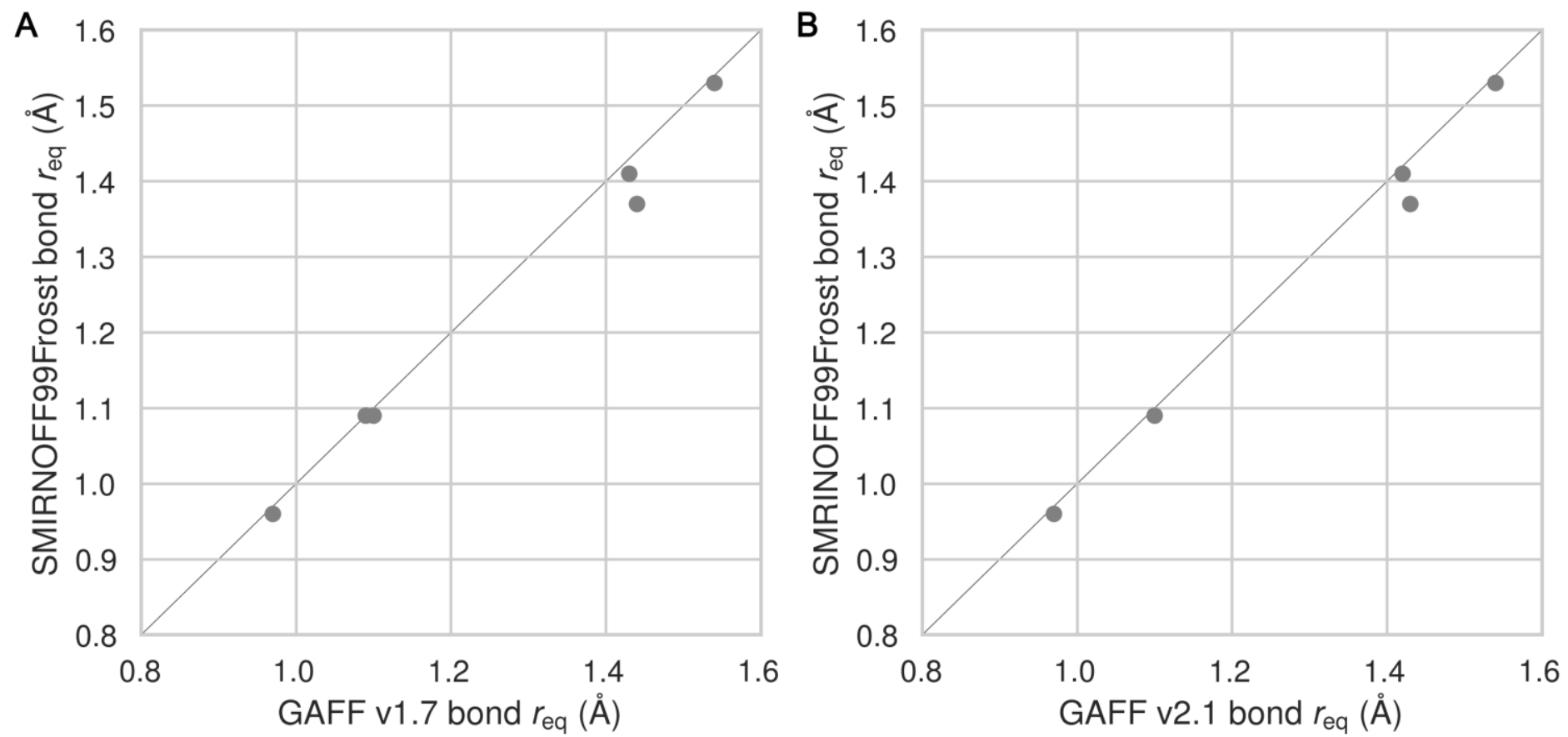

Figure S13: A comparison of bond equilibrium lengths for SMIRNOFF99Frosst, GAFF v1.7, and GAFF v2.1. Atom names refer to Figure 2.

Table S1: Experimental and predicted binding free energies $\left(\Delta G^{\circ}\right)$. Values in $\mathrm{kcal} / \mathrm{mol}$.

\begin{tabular}{lllllllll} 
System & Experimental & & SMIRNOFF99Frosst & & GAFF v1.7 & \multicolumn{3}{c}{ GAFF v2.1 } \\
\hline a-bam & Mean & SEM & Mean & SEM & Mean & SEM & Mean & SEM \\
a-but & -1.58 & 0.02 & -3.25 & 0.44 & -0.82 & 0.21 & -2.93 & 0.23 \\
a-cbu & -2.02 & 0.04 & -1.49 & 0.27 & -1.09 & 0.20 & -3.14 & 0.22 \\
a-chp & -2.51 & 0.02 & -1.33 & 0.19 & -0.89 & 0.22 & -3.73 & 0.21 \\
a-coc & -3.23 & 0.06 & -2.38 & 0.28 & -1.69 & 0.24 & -4.11 & 0.23 \\
a-cpe & -2.13 & 1.14 & -1.78 & 0.29 & -1.86 & 0.24 & -3.35 & 0.24 \\
a-ham & -3.53 & 0.02 & -1.59 & 0.25 & -1.50 & 0.29 & -3.79 & 0.22 \\
a-hep & -3.99 & 0.00 & -3.43 & 0.30 & -3.02 & 0.19 & -5.99 & 0.17 \\
a-hex & -3.38 & 0.01 & -3.95 & 0.21 & -3.93 & 0.20 & -6.23 & 0.17 \\
a-hp6 & -3.60 & 0.01 & -2.70 & 0.21 & -2.92 & 0.21 & -5.27 & 0.20 \\
a-hpa & -4.14 & 0.00 & -3.32 & 0.23 & -3.37 & 0.18 & -5.41 & 0.18 \\
a-hx2 & -3.34 & 0.00 & -3.02 & 0.32 & -3.16 & 0.22 & -6.03 & 0.22 \\
a-hx3 & -3.01 & 0.01 & -2.74 & 0.20 & -2.60 & 0.19 & -4.79 & 0.18 \\
a-mba & -1.76 & 0.01 & -2.39 & 0.25 & -1.58 & 0.23 & -3.94 & 0.23 \\
a-mha & -3.60 & 0.02 & -1.22 & 0.30 & -0.89 & 0.25 & -3.17 & 0.23 \\
a-mhp & -4.17 & 0.00 & -3.60 & 0.29 & -2.89 & 0.17 & -5.55 & 0.22 \\
a-nmb & -1.69 & 0.00 & -3.98 & 0.29 & -3.82 & 0.19 & -6.23 & 0.21 \\
a-nmh & -3.52 & 0.02 & -1.95 & 0.42 & -0.83 & 0.18 & -2.74 & 0.21 \\
a-oam & -4.61 & 0.01 & -4.15 & 0.59 & -2.92 & 0.18 & -5.56 & 0.19
\end{tabular}




$\begin{array}{lllllllll}\text { a-oct } & -4.62 & 0.02 & -4.64 & 0.30 & -4.85 & 0.24 & -6.81 & 0.19 \\ \text { a-pam } & -2.72 & 0.00 & -2.66 & 0.77 & -1.53 & 0.18 & -4.00 & 0.23 \\ \text { a-pnt } & -2.60 & 0.01 & -2.56 & 0.23 & -1.74 & 0.19 & -4.14 & 0.19 \\ \text { b-ben } & -1.64 & 0.02 & -2.85 & 0.62 & -1.83 & 0.29 & -2.45 & 0.17 \\ \text { b-cbu } & -1.55 & 0.17 & -1.88 & 0.20 & -1.64 & 0.36 & -2.77 & 0.17 \\ \text { b-chp } & -4.56 & 0.01 & -3.08 & 0.25 & -2.79 & 0.34 & -6.27 & 0.23 \\ \text { b-coc } & -4.97 & 0.04 & -3.28 & 0.23 & -3.36 & 0.26 & -7.13 & 0.22 \\ \text { b-cpe } & -3.05 & 0.01 & -3.57 & 0.34 & -3.55 & 0.31 & -5.93 & 0.27 \\ \text { b-ham } & -2.49 & 0.08 & -2.52 & 0.20 & -2.01 & 0.26 & -4.14 & 0.19 \\ \text { b-hep } & -3.39 & 0.18 & -3.41 & 0.28 & -3.34 & 0.35 & -4.15 & 0.23 \\ \text { b-hex } & -2.28 & 0.03 & -2.93 & 0.25 & -2.47 & 0.27 & -3.59 & 0.17 \\ \text { b-m4c } & -4.32 & 0.01 & -2.89 & 0.24 & -2.68 & 0.29 & -5.64 & 0.22 \\ \text { b-m4t } & -4.54 & 0.01 & -3.82 & 0.19 & -3.50 & 0.26 & -6.33 & 0.17 \\ \text { b-mch } & -4.18 & 0.01 & -3.69 & 0.22 & -3.31 & 0.26 & -6.07 & 0.17 \\ \text { b-mha } & -2.56 & 0.07 & -3.46 & 0.24 & -2.14 & 0.28 & -4.66 & 0.18 \\ \text { b-mo3 } & -2.16 & 0.01 & -2.87 & 0.38 & -2.73 & 0.41 & -2.79 & 0.20 \\ \text { b-mo4 } & -2.51 & 0.01 & -4.19 & 0.41 & -3.10 & 0.31 & -3.49 & 0.21 \\ \text { b-mp3 } & -1.46 & 0.04 & -3.03 & 0.27 & -2.48 & 0.28 & -3.00 & 0.19 \\ \text { b-mp4 } & -2.19 & 0.01 & -3.02 & 0.32 & -2.77 & 0.34 & -3.06 & 0.21 \\ \text { b-oam } & -3.59 & 0.12 & -3.35 & 0.28 & -2.60 & 0.30 & -5.25 & 0.23 \\ \text { b-pb3 } & -3.52 & 0.01 & -3.49 & 0.32 & -2.87 & 0.30 & -4.58 & 0.17 \\ \text { b-pb4 } & -3.60 & 0.02 & -3.62 & 0.33 & -3.34 & 0.35 & -4.71 & 0.23 \\ \text { b-pha } & -1.70 & 0.05 & -3.24 & 0.31 & -2.55 & 0.29 & -3.98 & 0.19 \\ \text { b-pnt } & -1.27 & 0.32 & -2.22 & 0.25 & -1.73 & 0.29 & -2.00 & 0.16\end{array}$

Table S2: Experimental and predicted binding enthalpies $(\Delta H)$. Values in $\mathrm{kcal} / \mathrm{mol}$.

System Experimental SMIRNOFF99Frosst

\begin{tabular}{lllllllll}
\hline & Mean & SEM & Mean & SEM & Mean & SEM & Mean & SEM \\
a-bam & -2.17 & 0.05 & -0.43 & 0.28 & -0.84 & 0.59 & -3.05 & 0.38 \\
a-but & -2.53 & 0.12 & -0.76 & 0.59 & -1.08 & 0.37 & -4.91 & 0.42 \\
a-cbu & -2.75 & 0.05 & -2.08 & 0.21 & -0.71 & 0.49 & -4.94 & 0.29 \\
a-chp & -2.99 & 0.23 & -3.42 & 0.39 & -2.33 & 0.28 & -5.27 & 0.35 \\
a-coc & -0.93 & 0.32 & -3.80 & 0.45 & -2.93 & 0.32 & -6.17 & 0.32 \\
a-cpe & -2.74 & 0.02 & -1.93 & 0.30 & -1.06 & 0.42 & -4.86 & 0.29 \\
a-ham & -4.19 & 0.02 & -4.02 & 0.33 & -2.33 & 0.30 & -6.91 & 0.29 \\
a-hep & -4.19 & 0.09 & -4.72 & 0.33 & -4.05 & 0.36 & -8.68 & 0.24 \\
a-hex & -3.40 & 0.02 & -4.33 & 0.30 & -2.95 & 0.30 & -7.43 & 0.31 \\
a-hp6 & -4.48 & 0.02 & -4.86 & 0.31 & -3.73 & 0.21 & -8.24 & 0.31 \\
a-hpa & -4.66 & 0.02 & -4.47 & 0.36 & -2.65 & 0.30 & -7.38 & 0.26
\end{tabular}




\begin{tabular}{|c|c|c|c|c|c|c|c|c|}
\hline$a-h \times 2$ & -4.12 & 0.06 & -4.24 & 0.31 & -2.35 & 0.32 & -6.56 & 0.30 \\
\hline a-hx3 & -3.36 & 0.05 & -2.25 & 0.55 & -2.80 & 0.32 & -5.51 & 0.28 \\
\hline$a-m b a$ & -2.68 & 0.07 & -0.95 & 0.41 & -0.32 & 0.37 & -3.11 & 0.36 \\
\hline a-mha & -4.28 & 0.02 & -3.31 & 0.50 & -2.16 & 0.25 & -6.40 & 0.34 \\
\hline a-mhp & -4.74 & 0.02 & -4.89 & 0.23 & -3.41 & 0.23 & -8.12 & 0.28 \\
\hline a-nmb & -2.57 & 0.06 & -1.10 & 0.28 & 0.03 & 0.23 & -3.34 & 0.27 \\
\hline a-nmh & -4.20 & 0.08 & -4.20 & 0.48 & -2.54 & 0.24 & -6.74 & 0.30 \\
\hline a-oam & -5.46 & 0.03 & -4.93 & 0.28 & -3.73 & 0.33 & -8.02 & 0.28 \\
\hline a-oct & -4.89 & 0.03 & -6.08 & 0.21 & -4.69 & 0.29 & -9.53 & 0.30 \\
\hline a-pam & -3.28 & 0.02 & -1.72 & 0.61 & -0.84 & 0.28 & -4.45 & 0.33 \\
\hline a-pnt & -2.75 & 0.01 & -2.05 & 0.37 & -1.62 & 0.33 & -5.99 & 0.30 \\
\hline b-ben & -2.51 & 0.08 & -0.45 & 0.56 & -0.76 & 0.82 & -1.30 & 0.26 \\
\hline b-cbu & 0.88 & 0.17 & 0.05 & 0.83 & -0.19 & 0.46 & 0.87 & 0.29 \\
\hline b-chp & -2.96 & 0.01 & 0.88 & 0.40 & 1.82 & 0.61 & -4.39 & 0.31 \\
\hline$b-c o c$ & -3.92 & 0.06 & 0.80 & 0.47 & 0.45 & 0.98 & -5.57 & 0.44 \\
\hline b-cpe & -1.09 & 0.01 & 1.86 & 0.37 & 3.62 & 0.65 & -1.32 & 0.30 \\
\hline b-ham & 0.60 & 0.05 & 1.66 & 0.68 & 2.29 & 0.78 & 0.42 & 0.33 \\
\hline b-hep & 0.42 & 0.04 & -0.05 & 0.38 & 1.91 & 0.29 & -0.92 & 0.27 \\
\hline b-hex & 1.31 & 0.04 & 0.41 & 0.65 & 1.30 & 0.60 & -0.21 & 0.27 \\
\hline b-m4c & -2.27 & 0.01 & 2.18 & 0.48 & 2.62 & 0.55 & -3.13 & 0.30 \\
\hline b-m4t & -2.17 & 0.02 & 1.26 & 0.45 & 2.49 & 0.51 & -3.11 & 0.29 \\
\hline b-mch & -2.29 & 0.03 & -0.79 & 1.08 & 2.27 & 0.73 & -3.37 & 0.34 \\
\hline b-mha & 0.47 & 0.03 & 0.53 & 0.55 & 2.48 & 0.64 & 0.28 & 0.29 \\
\hline b-mo3 & -2.93 & 0.03 & -2.66 & 0.46 & -0.59 & 0.45 & -2.50 & 0.28 \\
\hline b-mo4 & -1.96 & 0.01 & -2.69 & 0.58 & -0.91 & 0.33 & -3.05 & 0.29 \\
\hline b-mp3 & -2.75 & 0.13 & -1.09 & 0.68 & 0.68 & 0.48 & -2.64 & 0.31 \\
\hline b-mp4 & -2.89 & 0.05 & -2.84 & 0.76 & 0.78 & 0.60 & -2.34 & 0.28 \\
\hline b-oam & -0.48 & 0.03 & 0.98 & 0.41 & 2.66 & 0.43 & -0.52 & 0.34 \\
\hline b-pb3 & -2.25 & 0.01 & -1.59 & 0.94 & 1.78 & 0.36 & -2.24 & 0.30 \\
\hline b-pb4 & -2.82 & 0.01 & -0.02 & 0.62 & -1.44 & 0.78 & -3.70 & 0.29 \\
\hline b-pha & -1.79 & 0.11 & -1.10 & 0.69 & -1.34 & 0.97 & -3.45 & 0.36 \\
\hline b-pnt & 1.89 & 0.53 & -0.79 & 1.01 & -0.51 & 0.84 & 0.40 & 0.31 \\
\hline
\end{tabular}

Table S3: Experimental and predicted binding entropies $\left(-T \Delta S^{\circ}\right)$. Values in $\mathrm{kcal} / \mathrm{mol}$.

\begin{tabular}{lllllllll} 
System & Experimental & & \multicolumn{2}{c}{ SMIRNOFF99Frosst } & & GAFF v1.7 & & \multicolumn{2}{c}{ GAFF v2.1 } \\
\hline & Mean & SEM & Mean & SEM & Mean & SEM & Mean & SEM \\
a-bam & 0.59 & 0.05 & -2.82 & 0.53 & 0.02 & 0.63 & 0.11 & 0.45 \\
a-but & 1.02 & 0.13 & -0.73 & 0.65 & -0.01 & 0.42 & 1.77 & 0.47 \\
a-cbu & 0.73 & 0.05 & 0.75 & 0.28 & -0.17 & 0.54 & 1.21 & 0.36
\end{tabular}




\begin{tabular}{|c|c|c|c|c|c|c|c|c|}
\hline a-chp & 0.48 & 0.24 & 1.04 & 0.48 & 0.63 & 0.37 & 1.16 & 0.41 \\
\hline$a-c o c$ & -2.30 & 1.18 & 2.02 & 0.53 & 1.07 & 0.40 & 2.82 & 0.40 \\
\hline a-cpe & 0.61 & 0.03 & 0.33 & 0.39 & -0.44 & 0.51 & 1.07 & 0.37 \\
\hline a-ham & 0.66 & 0.02 & 0.59 & 0.44 & -0.68 & 0.36 & 0.92 & 0.34 \\
\hline a-hep & 0.20 & 0.09 & 0.77 & 0.39 & 0.12 & 0.41 & 2.45 & 0.29 \\
\hline a-hex & 0.02 & 0.02 & 1.62 & 0.37 & 0.03 & 0.36 & 2.17 & 0.37 \\
\hline a-hp6 & 0.88 & 0.02 & 1.54 & 0.38 & 0.37 & 0.27 & 2.84 & 0.36 \\
\hline a-hpa & 0.52 & 0.02 & 1.45 & 0.48 & -0.50 & 0.37 & 1.35 & 0.34 \\
\hline$a-h \times 2$ & 0.78 & 0.06 & 1.49 & 0.37 & -0.25 & 0.37 & 1.77 & 0.35 \\
\hline$a-h \times 3$ & 0.35 & 0.05 & -0.13 & 0.61 & 1.23 & 0.40 & 1.58 & 0.36 \\
\hline a-mba & 0.92 & 0.07 & -0.27 & 0.51 & -0.57 & 0.44 & -0.06 & 0.43 \\
\hline a-mha & 0.68 & 0.02 & -0.29 & 0.58 & -0.73 & 0.30 & 0.85 & 0.40 \\
\hline a-mhp & 0.57 & 0.02 & 0.92 & 0.37 & -0.41 & 0.30 & 1.89 & 0.35 \\
\hline a-nmb & 0.88 & 0.06 & -0.85 & 0.50 & -0.86 & 0.29 & 0.61 & 0.35 \\
\hline a-nmh & 0.68 & 0.08 & 0.05 & 0.76 & -0.38 & 0.30 & 1.18 & 0.36 \\
\hline a-oam & 0.85 & 0.03 & 0.25 & 0.57 & -0.60 & 0.37 & 1.02 & 0.34 \\
\hline a-oct & 0.27 & 0.04 & 1.44 & 0.37 & -0.16 & 0.38 & 2.72 & 0.36 \\
\hline a-pam & 0.56 & 0.02 & -0.94 & 0.98 & -0.68 & 0.34 & 0.45 & 0.40 \\
\hline a-pnt & 0.15 & 0.01 & -0.51 & 0.43 & -0.12 & 0.38 & 1.84 & 0.36 \\
\hline b-ben & 0.87 & 0.08 & -2.40 & 0.83 & -1.07 & 0.87 & -1.15 & 0.31 \\
\hline b-cbu & -2.43 & 0.24 & -1.93 & 0.85 & -1.45 & 0.58 & -3.64 & 0.34 \\
\hline b-chp & -1.60 & 0.01 & -3.96 & 0.47 & -4.61 & 0.69 & -1.87 & 0.39 \\
\hline$b-c o c$ & -1.05 & 0.07 & -4.08 & 0.53 & -3.81 & 1.02 & -1.56 & 0.49 \\
\hline b-cpe & -1.96 & 0.01 & -5.43 & 0.51 & -7.17 & 0.72 & -4.60 & 0.40 \\
\hline b-ham & -3.09 & 0.09 & -4.19 & 0.71 & -4.29 & 0.82 & -4.56 & 0.38 \\
\hline b-hep & -3.81 & 0.18 & -3.36 & 0.47 & -5.25 & 0.45 & -3.23 & 0.35 \\
\hline b-hex & -3.59 & 0.05 & -3.34 & 0.70 & -3.77 & 0.66 & -3.38 & 0.32 \\
\hline b-m4c & -2.05 & 0.01 & -5.07 & 0.54 & -5.29 & 0.62 & -2.51 & 0.37 \\
\hline b-m4t & -2.37 & 0.02 & -5.08 & 0.49 & -5.99 & 0.57 & -3.22 & 0.33 \\
\hline b-mch & -1.89 & 0.03 & -2.90 & 1.10 & -5.58 & 0.78 & -2.70 & 0.38 \\
\hline b-mha & -3.03 & 0.08 & -4.00 & 0.60 & -4.62 & 0.69 & -4.94 & 0.34 \\
\hline b-mo3 & 0.77 & 0.03 & -0.20 & 0.60 & -2.14 & 0.61 & -0.29 & 0.34 \\
\hline b-mo4 & -0.55 & 0.01 & -1.50 & 0.71 & -2.19 & 0.46 & -0.45 & 0.36 \\
\hline b-mp3 & 1.29 & 0.14 & -1.94 & 0.74 & -3.16 & 0.56 & -0.35 & 0.37 \\
\hline b-mp4 & 0.70 & 0.05 & -0.19 & 0.83 & -3.55 & 0.69 & -0.72 & 0.35 \\
\hline b-oam & -3.11 & 0.12 & -4.33 & 0.50 & -5.26 & 0.53 & -4.73 & 0.41 \\
\hline b-pb3 & -1.27 & 0.01 & -1.90 & 0.99 & -4.64 & 0.47 & -2.34 & 0.34 \\
\hline b-pb4 & -0.78 & 0.02 & -3.60 & 0.71 & -1.90 & 0.85 & -1.01 & 0.37 \\
\hline
\end{tabular}




$\begin{array}{lllllllll}\text { b-pha } & 0.09 & 0.12 & -2.14 & 0.76 & -1.21 & 1.01 & -0.52 & 0.41 \\ \text { b-pnt } & -3.16 & 0.62 & -1.43 & 1.04 & -1.22 & 0.89 & -2.40 & 0.35\end{array}$

Table S4: Predicted thermodynamic properties for SMIRNOFF99Frosst relative to experiment in $\mathrm{kcal} / \mathrm{mol}$, analyzed using MBAR.

\begin{tabular}{llllllllllllll} 
& & & RMSE & & MSE & & $R^{2}$ & \multicolumn{3}{c}{ Slope } & \multicolumn{3}{c}{ Intercept } \\
\hline$\Delta \mathrm{G}^{\circ}$ & SMIRNOFF99Frosst & 0.80 & {$[0.62,1.00]$} & -0.04 & {$[-0.28,0.20]$} & 0.67 & {$[0.22,0.65]$} & 0.53 & {$[0.33,0.73]$} & -1.45 & {$[-0.80,-2.07]$} & 0.48 & {$[0.32,0.62]$} \\
$\Delta H$ & SMIRNOFF99Frosst & 1.83 & {$[1.37,2.28]$} & 0.73 & {$[0.26,1.24]$} & 0.44 & {$[0.21,0.66]$} & 0.84 & {$[0.54,1.18]$} & 0.36 & {$[-0.53,1.47]$} & 0.52 & {$[0.34,0.68]$} \\
$-\mathrm{T} \Delta \mathrm{S}^{\circ}$ & SMIRNOFF99Frosst & 1.84 & {$[1.44,2.25]$} & -0.76 & {$[-1.26,-0.26]$} & 0.40 & {$[0.15,0.63]$} & 0.87 & {$[0.50,1.24]$} & -0.84 & {$[-1.33,-0.36]$} & 0.32 & {$[0.11,-0.50]$}
\end{tabular}

Table S5: Predicted thermodynamic properties for each force field relative to experiment on ammonium guests. Values in $\mathrm{kcal} / \mathrm{mol}$.

\begin{tabular}{|c|c|c|c|c|c|c|c|c|c|c|c|c|c|}
\hline & & RMSE & & MSE & & $\mathrm{R}^{2}$ & & Slope & & Intercept & & Tau & \\
\hline$\Delta G^{\circ}$ & SMIRNOFF99Frosst & 0.76 & {$[0.43,1.11]$} & -0.10 & {$[-0.54,0.31]$} & 0.48 & {$[0.07,0.84]$} & 0.69 & {$[0.19,1.16]$} & -1.06 & {$[0.54,-2.77]$} & 0.44 & {$[0.75,0.04]$} \\
\hline$\Delta \mathrm{G}^{\circ}$ & GAFF v1.7 & 0.77 & {$[0.59,0.95]$} & 0.69 & {$[0.51,0.88]$} & 0.90 & {$[0.76,0.98]$} & 1.08 & {$[0.88,1.26]$} & 0.95 & {$[1.56,0.32]$} & 0.74 & {$[0.91,0.50]$} \\
\hline$\Delta \mathrm{G}^{\circ}$ & GAFF v2.1 & 1.85 & {$[1.59,2.09]$} & -1.79 & {$[-2.04,-1.53]$} & 0.93 & {$[0.83,0.98]$} & 1.32 & {$[1.13,1.51]$} & -0.80 & {$[-0.20,-1.46]$} & 0.76 & {$[0.92,0.53]$} \\
\hline$\Delta \mathrm{H}$ & SMIRNOFF99Frosst & 1.15 & {$[0.77,1.51]$} & 0.83 & {$[0.39,1.27]$} & 0.89 & {$[0.76,0.97]$} & 1.15 & {$[0.89,1.53]$} & 1.31 & {$[2.81,0.38]$} & 0.78 & {$[0.92,0.56]$} \\
\hline$\Delta \mathrm{H}$ & GAFF v1.7 & 2.12 & {$[1.77,2.47]$} & 2.02 & {$[1.67,2.37]$} & 0.92 & {$[0.80,0.98]$} & 1.09 & {$[0.86,1.35]$} & 2.29 & {$[3.34,1.39]$} & 0.75 & {$[0.90,0.54]$} \\
\hline$\Delta \mathrm{H}$ & GAFF v2.1 & 1.90 & {$[1.31,2.43]$} & -1.51 & {$[-2.15,-0.88]$} & 0.96 & {$[0.91,0.99]$} & 1.54 & {$[1.38,1.83]$} & 0.09 & {$[1.18,-0.44]$} & 0.81 & {$[0.95,0.62]$} \\
\hline$-T \Delta S^{\circ}$ & SMIRNOFF99Frosst & 1.47 & {$[0.90,2.10]$} & -0.93 & {$[-1.59,-0.31]$} & 0.65 & {$[0.13,0.91]$} & 0.99 & {$[0.58,1.35]$} & -0.88 & {$[-0.09,-1.66]$} & 0.26 & {$[0.64,-0.26]$} \\
\hline$-\mathrm{T} \Delta \mathrm{S}^{\circ}$ & GAFF v1.7 & 1.45 & {$[1.14,1.79]$} & -1.33 & {$[-1.66,-1.00]$} & 0.88 & {$[0.18,0.97]$} & 1.04 & {$[-0.02,1.37]$} & -1.27 & {$[-0.55,-1.62]$} & 0.28 & {$[0.64,-0.21]$} \\
\hline$-\mathrm{T} \Delta \mathrm{S}^{\circ}$ & GAFF v2.1 & 1.04 & {$[0.67,1.40]$} & -0.27 & {$[-0.84,0.26]$} & 0.89 & {$[0.29,0.98]$} & 1.36 & {$[-0.53,1.66]$} & -0.12 & {$[1.16,-0.59]$} & 0.23 & {$[0.62,-0.26]$} \\
\hline
\end{tabular}

Table S6: Predicted thermodynamic properties for each force field relative to experiment on carboxylate guests. Values in $\mathrm{kcal} / \mathrm{mol}$.

\begin{tabular}{|c|c|c|c|c|c|c|c|c|c|c|c|c|c|}
\hline & & RMSE & & MSE & & $\mathrm{R}^{2}$ & & Slope & & Intercept & & Tau & \\
\hline$\Delta \mathrm{G}^{\circ}$ & SMIRNOFF99Frosst & 0.87 & {$[0.59,1.16]$} & -0.36 & {$[-0.74,-0.01]$} & 0.34 & {$[0.02,0.68]$} & 0.45 & {$[0.11,0.75]$} & -1.85 & {$[-0.91,-2.83]$} & 0.40 & {$[0.67,0.07]$} \\
\hline$\Delta \mathrm{G}^{\circ}$ & GAFF v1.7 & 0.68 & {$[0.49,0.88]$} & 0.03 & {$[-0.28,0.34]$} & 0.52 & {$[0.16,0.80]$} & 0.68 & {$[0.33,0.97]$} & -0.84 & {$[0.08,-1.75]$} & 0.53 & {$[0.76,0.23]$} \\
\hline$\Delta \mathrm{G}^{\circ}$ & GAFF v2.1 & 1.46 & {$[1.21,1.71]$} & -1.36 & {$[-1.61,-1.10]$} & 0.81 & {$[0.61,0.93]$} & 1.18 & {$[0.85,1.46]$} & -0.87 & {$[0.02,-1.74]$} & 0.72 & {$[0.87,0.54]$} \\
\hline$\Delta \mathrm{H}$ & SMIRNOFF99Frosst & 1.41 & {$[0.94,1.93]$} & 0.20 & {$[-0.43,0.84]$} & 0.53 & {$[0.20,0.79]$} & 0.83 & {$[0.40,1.53]$} & -0.14 & {$[2.12,-1.30]$} & 0.59 & {$[0.80,0.30]$} \\
\hline$\Delta \mathrm{H}$ & GAFF v1.7 & 1.95 & {$[1.34,2.55]$} & 1.24 & {$[0.55,1.93]$} & 0.47 & {$[0.13,0.78]$} & 0.79 & {$[0.32,1.49]$} & 0.82 & {$[3.10,-0.54]$} & 0.53 & {$[0.75,0.23]$} \\
\hline$\Delta \mathrm{H}$ & GAFF v2.1 & 2.43 & {$[1.75,3.06]$} & -1.73 & {$[-2.51,-0.96]$} & 0.69 & {$[0.49,0.85]$} & 1.40 & {$[0.99,2.29]$} & -0.66 & {$[2.15,-1.61]$} & 0.63 & {$[0.82,0.35]$} \\
\hline$-\mathrm{T} \Delta \mathrm{S}^{\circ}$ & SMIRNOFF99Frosst & 1.73 & {$[1.17,2.29]$} & -0.57 & {$[-1.32,0.16]$} & 0.29 & {$[0.02,0.61]$} & 0.62 & {$[0.16,1.09]$} & -0.68 & {$[0.05,-1.43]$} & 0.27 & {$[0.58,-0.09]$} \\
\hline$-\mathrm{T} \Delta \mathrm{S}^{\circ}$ & GAFF v1.7 & 2.07 & {$[1.35,2.76]$} & -1.22 & {$[-2.00,-0.46]$} & 0.29 & {$[0.00,0.67]$} & 0.63 & {$[-0.02,1.18]$} & -1.31 & {$[-0.58,-2.09]$} & 0.27 & {$[0.58,-0.09]$} \\
\hline$-\mathrm{T} \Delta \mathrm{S}^{\circ}$ & GAFF v2.1 & 1.46 & {$[1.12,1.77]$} & 0.37 & {$[-0.27,1.00]$} & 0.50 & {$[0.13,0.76]$} & 0.93 & {$[0.58,1.30]$} & 0.37 & {$[1.07,-0.34]$} & 0.37 & {$[0.67,-0.01]$} \\
\hline
\end{tabular}

Table S7: Predicted thermodynamic properties for each force field relative to experiment on cyclic alcohol guests. Values in $\mathrm{kcal} / \mathrm{mol}$.

\begin{tabular}{|c|c|c|c|c|c|c|c|c|c|c|c|c|c|}
\hline & & RMSE & & MSE & & $\mathrm{R}^{2}$ & & Slope & & Intercept & & Tau & \\
\hline$\Delta \mathrm{G}^{\circ}$ & SMIRNOFF99Frosst & 1.07 & {$[0.66,1.58]$} & 0.71 & {$[0.22,1.21]$} & 0.54 & {$[0.09,0.86]$} & 0.55 & {$[0.20,0.84]$} & -0.84 & {$[0.16,-2.09]$} & 0.44 & {$[0.75,0.02]$} \\
\hline$\Delta \mathrm{G}^{\circ}$ & GAFF v1.7 & 1.22 & {$[0.86,1.67]$} & 0.93 & {$[0.45,1.41]$} & 0.56 & {$[0.12,0.89]$} & 0.59 & {$[0.25,0.89]$} & -0.47 & {$[0.64,-1.77]$} & 0.47 & {$[0.78,0.05]$} \\
\hline$\Delta \mathrm{G}^{\circ}$ & GAFF v2.1 & 1.80 & {$[1.48,2.15]$} & -1.64 & {$[-2.04,-1.14]$} & 0.73 & {$[0.19,0.98]$} & 1.01 & {$[0.49,1.27]$} & -1.63 & {$[-0.67,-3.19]$} & 0.66 & {$[0.89,0.27]$} \\
\hline$\Delta \mathrm{H}$ & SMIRNOFF99Frosst & 2.88 & {$[1.99,3.68]$} & 1.66 & {$[0.21,3.03]$} & 0.09 & {$[0.00,0.44]$} & 0.07 & {$[-1.28,1.67]$} & -0.29 & {$[3.93,-4.06]$} & 0.09 & {$[0.56,-0.35]$} \\
\hline$\Delta \mathrm{H}$ & GAFF v1.7 & 3.63 & {$[2.67,4.47]$} & 2.66 & {$[1.13,4.07]$} & 0.10 & {$[0.00,0.57]$} & 0.12 & {$[-1.09,2.28]$} & 0.91 & {$[6.66,-2.47]$} & 0.14 & {$[0.60,-0.31]$} \\
\hline$\Delta \mathrm{H}$ & GAFF v2.1 & 2.08 & {$[1.18,3.16]$} & -1.64 & {$[-2.54,-0.91]$} & 0.54 & {$[0.00,0.97]$} & 1.08 & {$[-0.37,1.90]$} & -1.51 & {$[0.83,-5.50]$} & 0.46 & {$[0.89,-0.09]$} \\
\hline$-T \Delta S^{\circ}$ & SMIRNOFF99Frosst & 2.47 & {$[1.62,3.36]$} & -0.96 & {$[-2.22,0.52]$} & 0.40 & {$[0.00,0.93]$} & 1.18 & {$[-0.45,2.26]$} & -0.88 & {$[0.36,-3.60]$} & 0.30 & {$[0.71,-0.20]$} \\
\hline$-T \Delta S^{\circ}$ & GAFF v1.7 & 3.00 & {$[2.07,3.88]$} & -1.73 & {$[-3.14,-0.18]$} & 0.37 & {$[0.00,0.93]$} & 1.23 & {$[-0.38,2.48]$} & -1.59 & {$[-0.31,-4.23]$} & 0.29 & {$[0.71,-0.20]$} \\
\hline$-T \Delta S^{\circ}$ & GAFF v2.1 & 1.80 & {$[0.68,3.19]$} & -0.00 & {$[-0.98,1.27]$} & 0.48 & {$[0.00,0.97]$} & 1.13 & {$[-0.22,1.96]$} & 0.08 & {$[1.14,-1.79]$} & 0.46 & {$[0.82,-0.02]$} \\
\hline
\end{tabular}

Table S8: Dihedral parameter differences between SMIRNOFF99Frosst and GAFF v1.7. Atom names refer to Figure 2. NP: not present.

SMIRNOFF99Frosst GAFF v1.7

$\begin{array}{llllllll}\text { Atom 1 } & \text { Atom 2 } & \text { Atom 3 } & \text { Atom 4 } & \text { Per } & \text { Phase } & \text { Height }(\mathrm{kcal} / \mathrm{mol}) & \text { Height }(\mathrm{kcal} / \mathrm{mol}) \\ \mathrm{H} 1 & \mathrm{C} 1 & \mathrm{C} 2 & \text { O2 } & 1 & 0 & 0.25 & \mathrm{NP} \\ \mathrm{H} 1 & \mathrm{C} 1 & \mathrm{C} 2 & \mathrm{O} 2 & 3 & 0 & 0.00 & 0.16\end{array}$

Table S9: Dihedral parameter differences between SMIRNOFF99Frosst and GAFF v2.1, where one dihedral has fewer or more periodicity terms than the corresponding term in the other force field. Atom names refer to Figure 2. NP: not present.

SMIRNOFF99Frosst GAFF v2.1

\begin{tabular}{llllllll}
\hline Atom 1 & Atom 2 & Atom 3 & Atom 4 & Per & Phase & Height $(\mathrm{kcal} / \mathrm{mol})$ & Height $(\mathrm{kcal} / \mathrm{mol})$ \\
$\mathrm{C} 1$ & $\mathrm{C} 2$ & $\mathrm{O} 2$ & $\mathrm{HO} 2$ & 1 & 0 & 0.25 & $\mathrm{NP}$ \\
$\mathrm{C} 1$ & $\mathrm{C} 2$ & $\mathrm{O} 2$ & $\mathrm{HO} 2$ & 3 & 0 & 0.16 & 0.00 \\
$\mathrm{C} 1$ & $\mathrm{O} 5$ & $\mathrm{C} 5$ & $\mathrm{C} 4$ & 1 & 0 & $\mathrm{NP}$ & 0.00
\end{tabular}




\begin{tabular}{|c|c|c|c|c|c|c|c|}
\hline $\mathrm{C} 1$ & O5 & C5 & C4 & 2 & 0 & 0.10 & 0.16 \\
\hline $\mathrm{C} 1$ & O5 & C5 & C4 & 3 & 0 & 0.38 & 0.24 \\
\hline C1 & O5 & C5 & C6 & 1 & 0 & NP & 0.00 \\
\hline $\mathrm{C} 1$ & O5 & C5 & C6 & 2 & 0 & 0.10 & 0.16 \\
\hline $\mathrm{C} 1$ & O5 & C5 & C6 & 3 & 0 & 0.38 & 0.24 \\
\hline C2 & C1 & O5 & C5 & 1 & 0 & NP & 0.00 \\
\hline C2 & C1 & O5 & C5 & 2 & 0 & 0.10 & 0.16 \\
\hline C2 & C1 & 05 & C5 & 3 & 0 & 0.38 & 0.24 \\
\hline C2 & C3 & O3 & $\mathrm{HO} 3$ & 1 & 0 & 0.25 & NP \\
\hline C2 & C3 & O3 & $\mathrm{HO} 3$ & 3 & 0 & 0.16 & 0.00 \\
\hline C5 & C6 & 06 & $\mathrm{HO} 6$ & 1 & 0 & 0.25 & NP \\
\hline C5 & C6 & O6 & $\mathrm{HO} 6$ & 3 & 0 & 0.16 & 0.00 \\
\hline $\mathrm{H} 1$ & C1 & $\mathrm{C} 2$ & $\mathrm{O} 2$ & 1 & 0 & 0.25 & NP \\
\hline $\mathrm{H} 1$ & C1 & $\mathrm{C} 2$ & O2 & 3 & 0 & 0.00 & 0.16 \\
\hline O1 & C1 & $\mathrm{C} 2$ & $\mathrm{O} 2$ & 1 & 0 & $\mathrm{NP}$ & 0.02 \\
\hline 01 & C1 & $\mathrm{C} 2$ & $\mathrm{O} 2$ & 2 & 0 & 1.18 & 0.00 \\
\hline 01 & C1 & $\mathrm{C} 2$ & $\mathrm{O} 2$ & 3 & 0 & 0.14 & 1.01 \\
\hline $\mathrm{O} 2$ & C2 & $\mathrm{C} 1$ & O5 & 1 & 0 & NP & 0.02 \\
\hline $\mathrm{O} 2$ & $\mathrm{C} 2$ & C1 & O5 & 2 & 0 & 1.18 & 0.00 \\
\hline $\mathrm{O} 2$ & $\mathrm{C} 2$ & C1 & O5 & 3 & 0 & 0.14 & 1.01 \\
\hline O5 & C5 & $\mathrm{C} 6$ & O6 & 1 & 0 & $\mathrm{NP}$ & 0.02 \\
\hline O5 & C5 & $\mathrm{C} 6$ & O6 & 2 & 0 & 1.18 & 0.00 \\
\hline O5 & C5 & $\mathrm{C} 6$ & O6 & 3 & 0 & 0.14 & 1.01 \\
\hline $\mathrm{HO} 2$ & $\mathrm{O} 2$ & $\mathrm{C} 2$ & C3 & 1 & 0 & 0.25 & NP \\
\hline $\mathrm{HO} 2$ & $\mathrm{O} 2$ & $\mathrm{C} 2$ & C3 & 3 & 0 & 0.16 & 0.00 \\
\hline HO3 & O3 & C3 & C4 & 1 & 0 & 0.25 & NP \\
\hline HO3 & O3 & C3 & C4 & 3 & 0 & 0.16 & 0.00 \\
\hline
\end{tabular}

